\title{
ORDINARY MEANS IMPLY RECURRENT MEANS
}

\author{
BY R. V. CHACON
}

Communiçated by J. L. Doob, July 20, 1964

Introduction. Let $(X, \mathfrak{T}, \mu)$ be a $\sigma$-finite measure space, let $T$ be a positive linear operator from $L_{1}(X)$ to $L_{1}(X)$ whose norm is less than or equal to one. Let $\left\{w_{k}\right\}, k \geqq 1$, be a sequence of non-negative numbers whose sum is one and let $\left\{u_{k}\right\}, k \geqq 0$, be the sequence defined by $u_{n}=w_{1} u_{n-1}+\cdots+w_{n} u_{0}, u_{0}=1$. Set, for any pair of functions $f$ in $L_{1}(X)$ and $p$ in $L_{1}(X), p \geqq 0, Q_{n}(f, p)=Z_{n}(f) / Z_{n}(p), Z_{n}(g)$ $=\sum_{0}^{n-1} u_{k} T^{k} g$. Baxter, [2], [3] utilizing [6], has obtained the following result:

THEOREM 1. The ratios $Q_{n}(f, p)$ have a finite limit almost everywhere on the set where $p>0$.

The method of proof given by Baxter is a considerable and nontrivial application of the methods given in [4]. The theorem reduces to that of [4] if one takes $w_{1}=1, w_{k}=0, k \geqq 2$. The purpose of the present note is to show that the theorem of [4] yields Theorem 1 directly and in a stronger form. The stronger form of Theorem 1 gives convergence almost everywhere on the set where $\sum_{0}^{\infty} u_{k} T^{k} p>0$ and answers a question raised in [3]. Our proof is also sufficient to yield the theorem of [1] (see [7]).

1. Proof. Let $(I, R, m)$ be the measure space obtained by taking $I$ to be the positive integers, $R$ the Borel field of all subsets of $I$, and $m$ the measure given by $m(\{1\})=1$ and, for $i \geqq 2$, by

$$
\begin{array}{r}
m(\{i\})=1-w_{1}-\cdots-w_{i-1}, \quad \beta_{n}=w_{n} /\left(1-w_{1}-\cdots-w_{n-1}\right), \\
n \geqq 2, \beta_{1}=w_{1} .
\end{array}
$$

Let $P$ be the transformation of $L_{1}(I)$ to $L_{1}(I)$ defined by left multiplication by the matrix

$$
P=\left(\begin{array}{ccccc}
\beta_{1} & 1-\beta_{1} & 0 & 0 & \cdots \\
\beta_{2} & 0 & 1-\beta_{2} & 0 & \cdots \\
: & \vdots & \vdots & \vdots &
\end{array}\right) .
$$

We use $P$ to denote the transformation and the matrix and represent the elements of $L_{1}(I)$ as column vectors. It follows easily that $\|P\|=1$, 
that $P$ is positive and, setting $P^{n}=\left(p_{i j}(n)\right)$, that $u_{n}=p_{11}(n), n \geqq 0 .{ }^{1}$

Taking $(Y, \mathfrak{F}, \gamma)$ to be the direct product of $(I, \Re, m)$ and $(X, \mathfrak{T}, \mu)$ and $U$ the direct product of $P$ and $T$, it follows that $U$ is a positive linear operator from $L_{1}(Y)$ to $L_{1}(Y)$ and that the norm of $U$ is less than or equal to one. We may therefore apply the ratio theorem of [4] to $U$ with $\tilde{f}(y)=f(i, x)=\delta_{i 1} \cdot f(x), \tilde{p}(y)=p(i, x)=\delta_{i 1} \cdot p(x)$ to obtain Theorem 1 with convergence almost everywhere on the set where $\sum_{0}^{\infty} u_{k} T^{k} p>0$, since $U^{k} f(i, x)=p_{1 i}(k) T^{k} f(x), U^{k} p(i, x)$ $=p_{1 i}(k) T^{k} p(x)$ and $p_{11}(k)=u_{k}$.

\section{BIBLIOGRAPHY}

1. L. Baez-Duarte, An ergodic theorem of Abelian type, Abstract 64T-336, Notices Amer. Math. Soc. 11 (1964), 467.

2. G. Baxter, An ergodic theorem with weighted averages, J. Math. Mech. 13 (1964), 481-488.

3. - A general ergodic theorem with weighted averages, Abstract 64T-326, Notices Amer. Math. Soc. 11 (1964), 464.

4. R. V. Chacon and D. S. Ornstein, A general ergodic theorem, Illinois J. Math. 4 (1960), 153-160.

5. J. L. Doob, Renewal theory from the point of view of the theory of probability, Trans. Amer. Math. Soc. 63 (1948), 422-438.

6. A. Garsia and S. Sawyer, Remarks on the ergodic theorem with weighted averages (to appear).

7. G.-C. Rota, On the maximal ergodic theorem for Abel-limits, Proc. Amer. Math. Soc. 14 (1963), 722-723.

BROWN UNIVERSITY

${ }^{1}$ This is related to renewal theory. See [5] for a discussion of relevant facts. 